\title{
Are consumers ready to take part in the Pharmacovigilance System?-a Portuguese preliminary study concerning ADR reporting
}

\author{
Cristiano Matos $^{1}$ • Florence van Hunsel ${ }^{2}$ • João Joaquim ${ }^{1}$
}

Received: 27 January 2015 / Accepted: 12 May 2015

(C) Springer-Verlag Berlin Heidelberg 2015

\begin{abstract}
Background New pharmacovigilance legislation allows consumers to report adverse drug reactions (ADRs) directly to competent authorities in all European Union countries. Consumer reporting is available in Portugal since July 2012. In 2013, the National Pharmacovigilance System (SNF) had received 3461 spontaneous ADR reports, of which only $1.4 \%$ $(n=50)$ were from consumers. Consumer reporting could be one opportunity to reduce underreporting.

Aim The aim of this study was to describe the attitudes and knowledge of the general public regarding spontaneous reporting and the reasons and opinions that can influence consumers' ADR underreporting.

Methods A descriptive-correlational study was performed looking for consumers' attitudes and knowledge regarding spontaneous reporting. A 6-month survey was conducted from June to November 2013 in general adult consumers from a community pharmacy in Coimbra, Portugal, who used prescribed medicines or over-the-counter (OTC) drugs. Attitudes and opinions were surveyed by personal interview in a closedanswer questionnaire using a Likert scale. Questionnaires from healthcare professionals or incomplete ones were not considered. Data were analyzed using descriptive statistics, chi-square $\left(\chi^{2}\right)$ tests, and Spearman's correlation coefficients. Results One thousand eighty-four questionnaires were collected (response rate of $81.1 \%$ ) and 948 completed were
\end{abstract}

Cristiano Matos

cristiano.r.matos@gmail.com

1 Instituto Politécnico de Coimbra, Coimbra Health School-ESTESC, Farmácia, Coimbra, Portugal

2 Netherlands Pharmacovigilance Centre Lareb, 's-Hertogenbosch, the Netherlands selected for analysis. Of the respondents, $44.1 \%$ never heard about SNF. Younger people and those with a higher education were significantly more likely to be aware of SNF. Only one consumer had previously reported directly an ADR. Reporting ADRs indirectly through a healthcare professional (HCP) was preferred by $62.4 \%$. The main reasons for consumers reporting spontaneous ADR would be the severity of reactions (81.1\% agreed or strongly agreed) and worries about their situation (73.4\% agreed or strongly agreed). Only weak and moderate correlations were found between studied statements..

Conclusions Consumers are more likely to do spontaneous report about severe reactions or if they are worried about the symptoms. Tailored and proactive information on ADR reporting and educational interventions on consumers could increase the number of reports from consumers in Portugal.

Keywords Pharmacovigilance · Direct consumer' reporting · ADR reporting $\cdot$ Attitudes and knowledge

\section{Introduction}

Voluntary adverse drug reaction (ADR) reporting is one of the most versatile pharmacovigilance methods, because, among other advantages, it covers the entire population as well as all drugs throughout their commercial life [1], being also a method that provides the highest volume of information with relatively lower maintenance cost than other pharmacovigilance methods [2].

The new European legislation about pharmacovigilance that came into force last July 2012 [3] is the biggest change in the human medicine regulation in the European Union (EU) since 1995. The importance of direct consumer reporting has been highlighted by this new European legislation that guides member states to take all appropriate measures to encourage 